\title{
Optimisation of Sound Localisation for Emergency Vehicle Sirens through a Prototype Audio System
}

\author{
David Moore, Stephen Boslem, and Vassilis Charissis \\ Glasgow Caledonian University, School of Engineering and Computing, \\ Department of Computing and Creative Technologies, Cowcaddens Road, Glasgow, UK \\ J.D.Moore@gcu.ac.uk, sbosle10@caledonian.ac.uk, \\ v.charissis@gmail.com
}

\begin{abstract}
This paper examines the issues associated with the localisation of emergency vehicles. A combinatory warning system is then proposed that aims to provide drivers of both civilian and emergency vehicles with a different sequence of auditory cues as well as an in-cabin warning when an emergency vehicle is in the close vicinity. For the early testing of this hybrid alert system, we used the modelling techniques currently available to the UK emergency services in order to estimate the concurrent efficiency of the siren's auditory warnings.
\end{abstract}

Keywords: Road Safety, Sound Localisation, Warning Systems, Ambisonics, Spatial Audio.

\section{Introduction}

Emergency vehicle (EV) warning systems currently consist of three forms of alert: an audible warning, flashing lights and coloured bodywork markings. Out of the three, the most prominent system is the audible warning, which is typically based on a sweeping siren pattern. Early localisation of sirens by members of the public makes safer and swifter manoeuvring through traffic possible. However, for pedestrians and motorists alike, sirens can cause confusion, disorientation and possible danger if not reacted to within a timely manner. According to the Accident Statistics published by the British Department of Transport Road, the driver's inability to locate the incoming EV resulted in 7 fatalities and 1,226 casualties in the UK in 2008 [1].

Today, cars have improved soundproofing and are often equipped with powerful stereos. These factors combined with the general cacophony of modern urban life are enough to mask alert sounds, making siren recognition and accurate localisation challenging. Previous work has shown that high levels of background noise can significantly affect the average driver's ability to clearly define the position of the EV [2]. It has also been shown that the human inability to accurately locate the direction of an approaching EV is linked to the siren patterns and the limitations of the human auditory system [3].

As roads become increasingly busier it is important to design and implement systems that alert the driver of the proximity of incoming emergency vehicles and, more importantly, which direction they are approaching from. In this work we 
investigate the issues of localising EVs and in turn propose a new combinatory approach that provides the drivers with a different sequence of auditory cues as well as an in-cabin alert presented when an EV is in the close vicinity.

The section following looks at research into sound localisation as well as current siren localisation issues. Then the "Proposed System" section offers a succinct overview of the prototype combinatory approach. Then follows information on the modelling and simulation scenarios used to evaluate the effectiveness of the proposed system in contrast to the existing setup. The results are presented and then the conclusion section outlines the limitations and considerations of the proposed system and presents a plan outlining how we intend to develop our system in future work.

\section{Siren Localisation Issues}

\subsection{Sound Localisation}

Sound can be the first warning of events that are taking place around us. Unlike vision, which can be obscured by buildings and other objects, sound is perceptible from all directions and can travel around objects to some extent. Thus, auditory information is vital for situation awareness on the roads, both as a complement and a supplement to vision.

The human auditory system uses both binaural cues and monaural cues for determining the angle and distance of a sound source in the horizontal and vertical planes [4]. Binaural cues occur because we have two ears separated by the width of our head. Hence, any sound that originates from either side of our head will arrive at the ear closest to it before reaching the other ear; it will also be louder at the closest ear. These cues are known as Interaural Time Difference (ITD) and Interaural Level difference (ILD) respectively. ITDs are not an effective cue at high frequencies because certain time delays may result in the same phase difference at the ears. Conversely, ILDs are not effective at low frequencies because a sound's wavelength is larger than the diameter of the head resulting in little or no attenuation by the head when en route to the furthest ear.

Although ITD and ILD are known to be the major cues for sound localisation they are not enough on their own for localising sounds. For sound sources in the horizontal plane there are always two points around the listener with identical ITDs and ILDs. For example, sound arriving from a source at $45^{\circ}$ from the front in the horizontal plane will have an identical ITD and ILD as sound arriving from a source at $135^{\circ}$ from the front. If the vertical plane is considered as well then there will be a whole series of points on the surface of a cone that have the same ITD and ILD. This is known as the cone of confusion (see Fig.1).

To resolve this ambiguity spectral cues are used which occur as a result of the directional-dependent filtering caused by sound reflecting off the ear's pinnae and upper body. A number of different studies have demonstrated that monaural (single ear) spectral cues are vital for the localisation of sound sources above and below the listener $[5,6]$. This has been clearly demonstrated in experiments by Gardner and Gardner [7]. There is also substantial evidence that the spectral cues incurred because of sound reflecting off the pinnae help us discriminate sounds coming from the front and back [8-10]. 


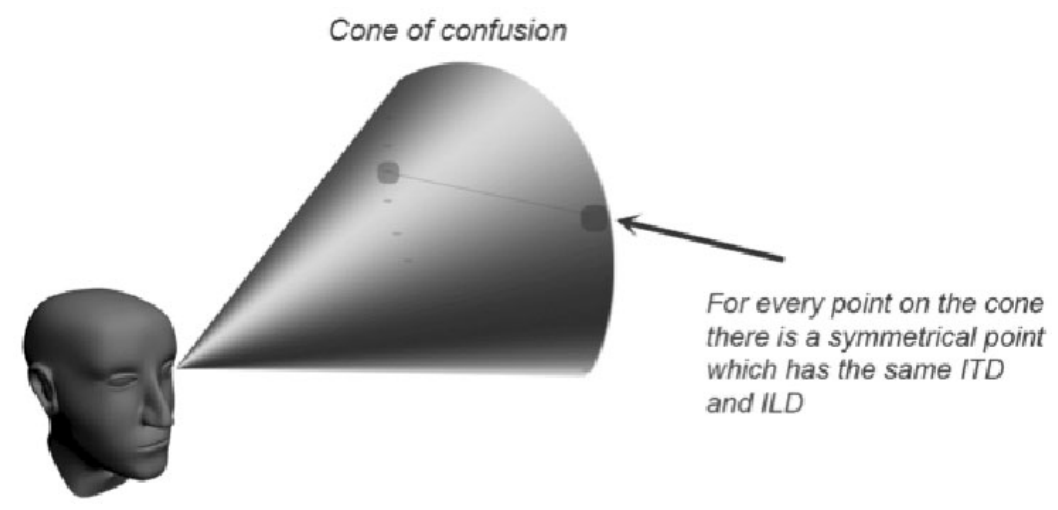

Fig. 1. The cone of confusion

Numerous studies have shown that human localisation accuracy varies markedly with frequency $[11,12]$. Generally, human localisation accuracy remains approximately constant for frequencies below $1 \mathrm{kHz}$. For frequencies between about $1 \mathrm{kHz}$ and $3 \mathrm{kHz}$, however, acuity degrades somewhat until after $3 \mathrm{kHz}$ when it improves again. The reason for degradation in this frequency region is because interaural phase cues start to become ambiguous after $1 \mathrm{kHz}$, whereas below $3 \mathrm{kHz}$ the interaural level differences are not always significant enough for a listener to lateralise a sound successfully. This problematic cross-over region can be seen in various studies $[4,12]$.

Localisation has been shown to be most accurate directly in front of the listener [4]. This accuracy decreases as the source moves to the side of the listener and improves again at the direct rear. The relationship between the angle of the sound source and accuracy of localisation is approximately the same for both low and high frequencies. When the sound source is moving (as in the case of an approaching EV) this can add greater complexity to the task of sound localisation. Research has shown that humans can be quite inaccurate when determining the absolute location of a moving sound at a particular time [13,14].

\subsection{Existing Siren Issues}

All of the above physiological issues have a direct impact on the driver's interaction with emergency vehicles. A siren can only be accurately localised if it contains sufficient localisation cues and these cues are audible in the environment in which the source is produced. Existing sirens typically employ a sweeping pattern with the majority of sound energy in the region of $500 \mathrm{~Hz}$ to $1500 \mathrm{~Hz}$ - this can clearly be seen in the time-frequency plot provided below (see Fig. 2). However, this pattern is not necessarily the easiest source to localise. Sounds of greater than $500 \mathrm{~ms}$ duration, with broadband spectral content and strong onsets are known to be easier to localise accurately than narrowband or tonal sounds with gradual onsets [15]. 


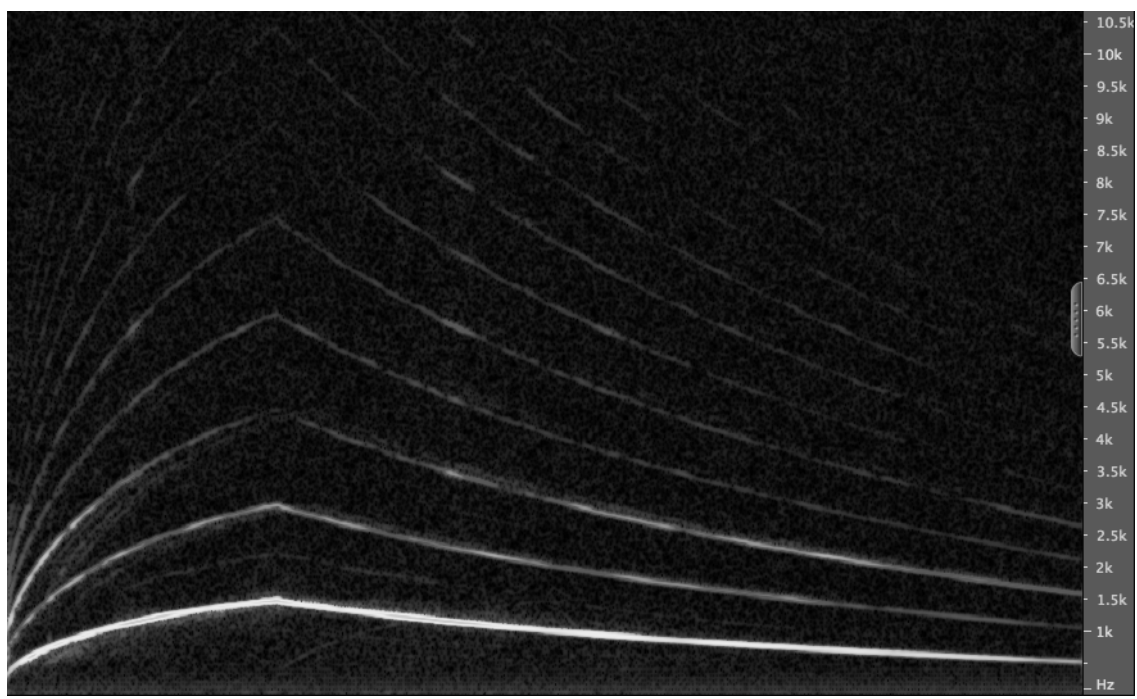

Fig. 2. Typical sweeping siren pattern

A previous investigation into audible warning systems showed that sirens were used $86 \%$ of the time by EVs when on call, but this was not always in unison with warning lights [16]. In a survey that examined public reactions to audible warning the results showed that nearly one third of drivers failed to detect the approaching emergency vehicle until it was less than 50m away. Even more astoundingly, $25 \%$ of the participants were completely unable to hear the audible warning systems on approach of the emergency vehicle. These figures again reinforce the limited effectiveness of current emergency vehicle audible warning systems [16, 17].

The aforementioned observations have clearly stated that some drivers could not differentiate the siren sound from the ambient noise at these distances, and evidently in some cases existing sirens are unable to overcome the background noise generated by driving on modern road networks, rendering them completely unusable in some cases [17-19]. Of all interactions with emergency vehicles, more than half of participants had to manoeuvre to let the vehicle pass. This discovery led to the so-called 'wake effect', where one third of these manoeuvres placed a third party at risk [20, 21].

\section{Proposed System}

The first improvement aims to minimise or even eliminate spatial ambiguity inherent in existing frequency siren tones. Therefore one of the siren patterns that we use in this study is designed to produce short, wide-band noise bursts that are known to be easier sources to localise than single frequency sweeps. Earlier investigations suggested that signals that possess relatively few harmonics are less resistant to masking, and they also need to be presented at much higher signal-to-noise ratio than signals that are acoustically richer [22, 23]. 
Adhering to this observation, siren patterns should contain the broadest audible frequency range possible in a range between $20 \mathrm{~Hz}$ to $20 \mathrm{kHz}$. Notably the main sweep of existing siren patterns only covers roughly the region between $500 \mathrm{~Hz}$ to $1500 \mathrm{~Hz}$, therefore it is significantly more difficult for both motorists and pedestrians to accurately localise the source and direction of the sound [3, 17]. However the human hearing has a peak in sensitivity which reaches approximately $3500 \mathrm{~Hz}$ to $4000 \mathrm{~Hz}$. To take account of the ear's increased sensitivity to this particular frequency band our proposed system uses a broader frequency bandwidth with a main sweep ranging from $400 \mathrm{~Hz}$ to $4000 \mathrm{~Hz}$. It is proposed that by expanding the frequency content of existing patterns into the region of 3500 to $4000 \mathrm{~Hz}$ will make them more obvious, yet still sound familiar to motorists and pedestrians, building on the existing learned association of these tones.

The second element of the proposed solution aims to reduce the problems associated with environmental noise and the secluded shell of the vehicle interior. It consists of an in-cabin auditory and visual warning system for informing the driver when an emergency vehicle is in the vicinity. Nowadays, nearly all car audio reproduction systems consist of at least 4 loudspeakers arranged in a rectangle shape (see Fig. 3). This configuration is well suited to presenting auditory information at different spatial positions around the driver.

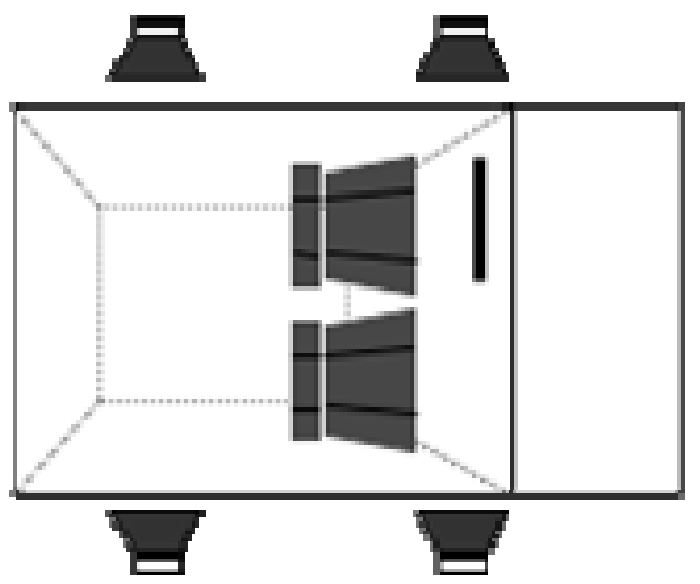

Fig. 3. Typical car loudspeaker layout

Therefore, we propose to relay a warning sound to the driver in the same direction as the approaching EV in order to reinforce its position. To do this, a surround sound technique known as Ambisonics will be employed to position the alert sound in the desired location. Ambisonics is currently an active topic of audio research and could be easily integrated with existing car audio systems. As the driver is not normally equidistant from the loudspeakers we intend to incorporate previous research by one of the authors that optimises the auditory localisation performance of Ambisonic surround sound for off-centre listening positions [24]. This second addition to the proposed system has been deemed essential for drivers that cannot hear and locate even the 
improved siren as has been highlighted. Several methods for automatically locating the EV before relaying a warning will be considered - see for example [25 - 27].

This two-part system aims to alert the driver simultaneously externally and internally to the EV. The leading vehicles in the traffic stream will then have a higher probability of identifying the alert and manoeuvre accordingly in order to support the safe passage of the EV. The preliminary experiments in the simulated environment demonstrated that a $30 \%$ speed increase could potentially be achieved with the combinatory proposed system. The predicted impact of increased road speeds on fatality rates and turn out times was examined using a simulation modelling method based on the FSEC modelling system. This is further discussed in the following section of the paper.

\section{Modeling and Simulation}

For the evaluation of the proposed solution it was deemed necessary to reproduce the effect of the system in a simulated environment and in turn contrast it to the impact of the typical auditory warning produced by the EVs. In this particular case, the Fire and Rescue Services of Scotland kindly provided the required information of current siren system. Furthermore we employed the official computer modelling methodology, which is used by the Central Scotland Fire and Rescue Services Fire Service Emergency Cover (FSEC), in order to achieve the validity of our comparative study.

This simulation, offered the comfort of experimenting in a safe, controlled environment. The simulation entailed different simulation scenarios typically modelled following real-life emergency situations which require the acute mobilisation of the Fire-brigade vehicular units. Notably the degree of realism embedded in these scenarios and factors that have been taken into account were crucial for the successful replication of reality in the simulation software.

In particular the base-cases, adhering to the standard speeds on the road network and consequently offer a truthful representation of the average road speeds typically attained by EVs with the use of the contemporary sirens. The main measurable data in this experiment was the distance that a fire-brigade vehicle can cover in a time defined array of 8 minutes in the selected region with centre the town of Falkirk. In turn the software measured the average speed achieved by the vehicles. The aforementioned two collected type of data could in turn define precisely the distribution of map coverage with and without the utilisation of the prototype system. Notably in the modelling process of the proposed system we included both the effect of the broadband noise patterns and RDS warning system in the simulation scenarios. This was feasible by utilising an existing trait of the FSEC software that allows the modification of individual road speeds, based on additional information.

As such we achieved to simulate the end-result of our system by predicting the increase in average speed in the specific road network. In turn both models were simulated and tested in the predefined scenarios with the use of the FSEC. The produced results were in turn visualised and propagated on the map, offering a visible differentiation on the EVs coverage with the use of both systems as shown in Figure 4 which illustrates the Ordnance Survey (OS) mapping in four distinctive map distributions varying from the standard road speed up to $30 \%$ increased speeds. 

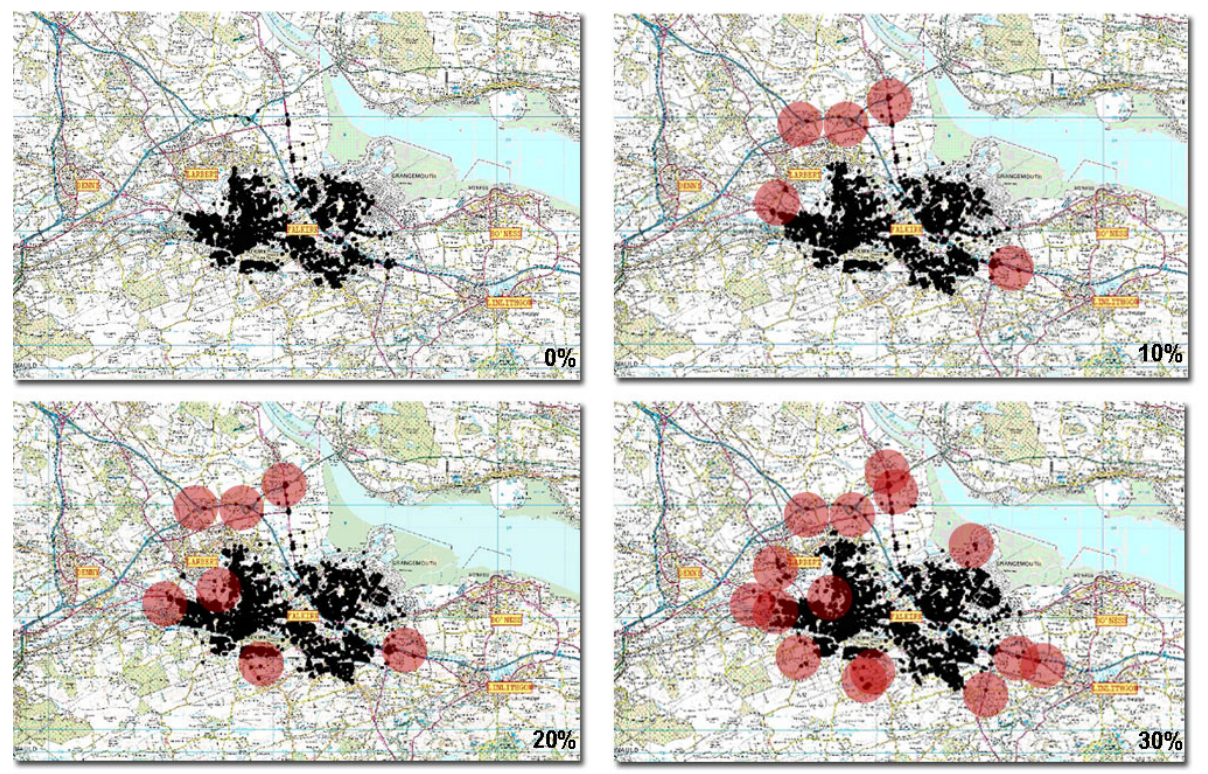

Fig. 4. Falkirk Station distance coverage in 8 minutes, under standard road speeds, $10 \%$, $20 \%$ and $30 \%$ increased speed respectively

\section{Discussion}

It is evident that in the infinitesimal time-limit of 8 minutes, the EVs can extend their action significantly with the use of the proposed system, covering remote areas which previously were impossible to reach urgently.

However it could be argued that this method could be in expense of the civilians' safety as the EVs accelerate significantly more in order to achieve these distances in the same timeframe. This potential hazard can be decreased considerably as the proposed system does not inform the driver only through the external auditory avenue (i.e. siren), but presents the information also inside the vehicle with the use of the RDS. To this point the RDS can offer also visual cues provided in the instrumentation panel of the vehicle or through a Head-up Display (HUD) system. The latter could convey the information in driver's field of view, attracting instantly the user's attention. This could be utilised as a supplementary warning method in case of auditory cues' inability to command user's awareness.

Except the quantitative evaluation through the simulation that offers a clear view of the potential outcomes we also performed a qualitative study aiming to reveal the EVs' drivers feedback for the proposed system which will not be analysed in this paper. Nonetheless an important issue was raised as a direct result of using questionnaires to gather the opinions and experiences of this specific group of user, regarding the subject of driver behaviour towards emergency vehicle situations. Interestingly the emergency vehicle drivers stated that even if audible warning systems were substantially improved, “...some motorists will still panic and not know 
what to do, whereas others will simply not want to give way." As such a small segment of the driving population will still fail to handle the situation in a safe fashion even if an improved audible warning system can alert motorists to approaching emergency vehicles at an earlier stage than previously possible. The specific issue can be potentially resolved or minimised with the use of a combinatory approach of warning interfaces that can increase gradually, yet substantially, the alert levels (i.e. auditory, visual and tactile) depending on the distance difference from the $\mathrm{EV}$ and the approach speed.

\section{Conclusions}

This paper offered a succinct description of a prototype audio system which optimises effectively the sound localisation of emergency vehicles. The system entails an experimental sound sequence for the siren used in conjunction to an RDS warning signal. The proposed system aims to increase drivers' awareness regarding the incoming emergency vehicle and provide sufficient sound localisation that will enable the driver to respond promptly. In turn the successful provision of road space to the emergency vehicles will result in larger area coverage and faster service from the acute mobilisation units. The evaluation of the proposed system in contrast to the typical siren systems used in fire-brigade vehicles presented a potential benefit of $30 \%$ in the increase of the EVs speeds. This amplification of vehicular speed has a direct effect in a significantly expanded area that the emergency services can reach in the typical 8 minute window. The quantitative methods used for the evaluation were based on the modelling and simulation software used officially by the emergency services in Scotland.

On our future work we endeavour to test exhaustively the proposed system in a number of other simulation scenarios which will factor in different weather conditions, traffic flows and simultaneous accidents that require a predictive model of optimised navigation system. Furthermore we intent to embed the auditory cues of the proposed system on our existing full-windshield Head-Up-Display interface in order to provide the civilian drivers with a number of possible warnings depending on the level of emergency in each case.

\section{References}

1. Department for Transport UK, http://www.dft.gov.uk/pgr/statistics/ datatablespublications/accidents/

2. Withington, D.J., Patterson, S.E.: Safer Sirens. Fire Engineers Journal 48, 6-10 (1998)

3. Withington, D.J.: The quest for better Ambulance Sirens. Ambulance UK 11, 20-21 (1996)

4. Blauert, J.: Spatial Hearing: The Psychophysics of Human Sound Localization. MIT Press, Cambridge (2001)

5. Wright, D., Hebrank, J.H., Wilson, B.: Pinna Reflections as Cues for Localization. The Journal of the Acoustical Society of America 56(3), 957-962 (1974) 
6. Moore, B., Oldfield, S.R., Dooley, G.J.: Detection and Discrimination of Spectral Peaks and Notches at $1 \mathrm{kHz}$ and $8 \mathrm{kHz}$. The Journal of the Acoustical Society of America 85(2), 820-836 (1989)

7. Gardner, M.B., Gardner, R.S.: Problem of Localization in the Median Plane: Effect of Pinnae Cavity Occlusion. The Journal of the Acoustical Society of America 53(2), 400408 (1973)

8. Kistler, D.J., Wightman, F.L.: A model of head-related transfer functions based on principal components analysis and minimum-phase reconstruction. The Journal of the Acoustical Society of America 91(3), 1637-1647 (1992)

9. Langendijk, E.H.A., Bronkhorst, A.W.: Contribution of spectral cues to human sound localization. The Journal of the Acoustical Society of America 112(4), 1583-1596 (2002)

10. Zahorik, P.: Perceptual Recalibration in Human Sound Localization: Learning to Remediate Front-Back Reversals. The Journal of the Acoustical Society of America 12(1), 343-359 (2006)

11. Stevens, S.S., Newman, E.B.: The Localization of Actual Sources of Sound. The American Journal of Psychology 48(2), 297-306 (1936)

12. Moore, B.: An Introduction to the Psychology of Hearing. Academic Press, London (2003)

13. Perrott, D.R., Musicant, A.D.: Minimum Auditory Movement Angle - Binaural Localization of Moving Sound Sources. Journal of the Acoustical Society of America 62, 1463-1466 (1977)

14. Grantham, D.W.: Detection and Discrimination of Simulated Motion of Auditory Targets in the Horizontal Plane. Journal of the Acoustical Society of America 79, 1939-1949 (1986)

15. Vliegen, J., Optstal, A.J.V.: The Influence of Duration and Level on Human Sound Localization. Journal of the Acoustical Society of America 115, 1705-1713 (2004)

16. Saunders, G., Gough, A.: Emergency Ambulances on the Public Highway Linked with Inconvenience and Potential Danger to Road Users. Emergency Medical Journal 20, 277 280 (2003)

17. Ho, C., Spence, C.: Assessing the effectiveness of various auditory cues in capturing a driver's visual attention. Journal of experimental psychology: Applied 11, 157-174 (2005)

18. Lenné, M.G., Triggs, T.J., Mulvihill, C.M., Regan, M.A., Corben, B.F.: Detection of emergency vehicles: Driver response to advance warning in a driving simulator. Human Factors 50, 135-144 (2008)

19. Llaneras, R.E., Green, C.A., Kiefer, R.J., Chundrlik, W.J., Atlan, O.D., Singer, J.P.: Design and evaluation of a prototype rear obstacle detection and driver warning system. Human Factors 47, 199-215 (2005)

20. Xiao, D.-1., Tian, Y.-j.: Reliability of Emergency Rescue System on Highway. In: Proceedings of Second International Conference on Intelligent Computation Technology and Automation (ICICTA 2009), Changsha, Hunan, China, vol. 2, pp. 564-567 (2009)

21. Sherratt, R.S.: Results of a DSP based adaptive cancellation filter system for the chirp, pulsar and localiser sirens. In: IEEE Nordic Signal Processing Symposium (NORSIG 2000), KolmArden, pp. 235-238 (June 2000)

22. Wightman, F.L., Kistler, D.J.: Sound localisation. In: Human Psychophysics, pp. 155-192. Springer, New York (1993)

23. Wenzel, E.M., Arruda, M., Kistler, D.J., Wightman, F.L.: Localisation using nonindividualised head-related transfer functions. Journal of the Acoustic Society of America 94, 111-123 (1993) 
24. Moore, J.D., Wakefield, J.P.: Optimisation of the Localisation Performance of Irregular Ambisonic Decoders for Multiple Off-Centre Listeners. Presented at the 128th Audio Engineering Society Convention, London, UK (2010)

25. Valin, J.M., Michaud, F., Rouat, J.: Robust localisation and tracking of simultaneous moving sound sources using beam forming and particle filtering. In: Proceedings of the IEEE International Conference on Acoustics, Speech and Signal Processing, ICASSP (2006)

26. Atmoko, H., Tan, D.C., Tian, G.Y., Fazenda, B.: Accurate Sound Source Localization in a Reverberant Environment using Multiple Acoustic Sensors. Journal of Measurement and Technology 19(2) (2008)

27. Fazenda, B., Atmoko, H., Gu, F., Guan, L., Ball, A.: Acoustic Based Safety Emergency Vehicle Detection for Intelligent Transport Systems. In: Proceedings of the ICROS-SICE International Joint Conference Fukuoka, Japan, pp. 4250-4255 (2009)

28. Charissis, V., Papanastasiou, S., Vlachos, G.: Interface Development for Early Notification Warning System: Full Windshield Head-Up Display Case Study. In: Jacko, J.A. (ed.) HCII 2009. LNCS, vol. 5613, pp. 683-692. Springer, Heidelberg (2009) ISBN 978-3-64202582-2 